Cultures \& Conflits

53 | printemps 2004

Surveillance politique : regards croisés

\title{
Surveiller et faire élire : surveillance politique et pratiques de la
}

\section{Christophe Voillot}

\section{(2) OpenEdition \\ 1 Journals}

\section{Édition électronique}

URL : http://journals.openedition.org/conflits/995

DOI : $10.4000 /$ conflits.995

ISSN : $1777-5345$

Éditeur :

CCLS - Centre d'études sur les conflits lilberté et sécurité, L'Harmattan

\section{Édition imprimée}

Date de publication : 1 mars 2004

Pagination : 71-82

ISBN : 2-7475-6791-5

ISSN : 1157-996X

\section{Référence électronique}

Christophe Voillot, "Surveiller et faire élire : surveillance politique et pratiques de la », Cultures \& Conflits [En ligne], 53 | printemps 2004, mis en ligne le 04 octobre 2004, consulté le 30 mars 2021.

URL : http://journals.openedition.org/conflits/995 ; DOI : https://doi.org/10.4000/conflits.995

Ce document a été généré automatiquement le 30 mars 2021.

Creative Commons License 


\title{
Surveiller et faire élire : surveillance politique et pratiques de la
}

\author{
Christophe Voillot
}

La coïncidence apparente entre l'intensification de la «surveillance politique » et l'extension du suffrage peut être appréhendée au travers de l'analyse de la constitution d'enjeux électoraux spécifiques dans la France du XIXème siècle. Néanmoins, parler de "surveillance politique » à propos des élections ne va pas de soi et pose un ensemble de problèmes que je souhaite évoquer de manière liminaire.

L'adjectif «politique » désigne rétrospectivement un ensemble d'activités dont l'autonomisation progressive au XIXème siècle peut nourrir toute une série d'anachronismes, plus ou moins incongrus, sous la plume d'un commentateur contemporain. Plutôt qu'une "surveillance politique ", il faudrait envisager de prime abord ce que pourrait être, ce qu'a pu être et devenir, une surveillance d'activités politiques et, dans le même mouvement, se demander ce qui est effectivement considéré comme " politique » à un moment donné et dans un lieu donné, y compris et surtout s'agissant des opérations électorales. En effet, l'identification comme activité politique de ces dernières n'est pas séparable de la mise en oeuvre et de la mise en forme de "technologies d'Etat» qui vont faire de la nomination élective un moyen d'accès à certaines positions de pouvoir. Elle n'est pas séparable non plus de la constitution - à partir notamment de cette "science politique balbutiante " que contiennent, selon Alain Corbin, les rapports des administrateurs du XIXème siècle ${ }^{1}$ d'un savoir spécialisé dont les schèmes de perception déterminent aujourd'hui la manière dont le politiste identifie et marque ce qu'il croit être « ses » objets ${ }^{2}$.

Dans ces conditions, parler au présent de «surveillance politique », c'est d'une certaine manière enregistrer le résultat d'un processus, celui d'une monopolisation progressive des activités de surveillance, partie intégrante d'un processus étatique de monopolisation de la violence collective. Sous réserve d'évolutions récentes liées à la remise en cause de l'autonomie des Etats parlementaires nationaux ${ }^{3}$, nous avons donc de bonnes raisons d'identifier, sur le mode du sens commun, surveillance, police et maintien de l'ordre public. Il n'en a peut-être pas toujours été ainsi... 
Enfin, il est un point commun, pour ne pas dire un point aveugle, entre opérations électorales et activités de surveillance. Dans les deux cas, nous avons à faire à des opérations mobilisant ponctuellement mais de manière structurée et centralisée des agents de l'Etat, en priorité ceux des ministères de l'Intérieur et de la police. Il y a donc quelque légitimité à vouloir étudier ensemble activités de surveillance et pratiques électorales, et à le faire à travers l'étude des pratiques et des répertoires d'action communs à ces deux domaines d'activité.

A travers l'analyse du rôle et de la place des activités de surveillance dans les pratiques de la " candidature officielle " ${ }^{4}$, il est possible d'appréhender plus concrètement cette notion de "surveillance politique ». Cette surveillance se donne ici à voir dans une conjoncture spécifique : la « crise » des années 1815-1816 et la dissolution de la fameuse "Chambre introuvable " . Autour de cette dissolution, et des élections générales qui s'en suivent, apparaît en effet un « circuit de surveillance » dont j'analyserai la mise en place et le fonctionnement puis les transformations au cours des premières années de la Seconde Restauration.

La mise en place d'un " circuit de surveillance "

Un double impératif structure les conduites des acteurs impliqués dans la, ou plutôt les, compétitions électorales de 1816 : premièrement, «faire voter »en faveur du ou des candidats de son camp; deuxièmement, prévenir et contrecarrerles activités du camp adverse. C'est à partir de ce double impératif qu'apparaît toute une gamme de pratiques dont la plupart ne sont que la transposition à une situation électorale particulière de pratiques inscrites auparavant dans des logiques de situation différentes, et donc issues d'expériences passées distinctes d'acteurs engagés dans les luttes pour l'obtention de charges électives. Le deuxième impératif est d'ailleurs intrinsèquement et pratiquement lié au premier : ce sont les peurs suscitées chez ceux qui estiment avoir des enjeux, personnels ou collectifs, dans les nominations à effectuer qui suscitent des actions visant à neutraliser les activités tactiques du camp adverse. A partir de quelques exemples significatifs, tirés d'une enquête dans les archives ${ }^{6}$, je tenterai de restituer ces enjeux et les pratiques associées aux opérations électorales de l'automne 1816, en mettant en exergue celles constitutives d'un «circuit de surveillance ».

Qu'entend-on par « circuit de surveillance »? Il s'agit en l'occurrence de l'ensemble des agents mobilisés par le ministère de la police générale ${ }^{7}$, dont le titulaire Decazes est un des promoteurs de la dissolution de la Chambre des députés ${ }^{8}$, pour surveiller, premièrement, les activités des camps adverses (les «jacobins» d'une part, et les " ultraroyalistes » partisans du comte d'Artois de l'autre) et, deuxièmement, la loyauté des soutiens aux candidats officiels qui, pour l'essentiel, sont les présidents des collèges électoraux de département désignés par ordonnance royale. La notion de circuit renvoie plus directement à la manière dont ces agents travaillent. Les informations recueillies dans les différents départements sont centralisées, une synthèse de la correspondance est effectuée dans les bureaux du ministère, et ces informations servent de base aux instructions données ensuite aux agents chargés soit de la promotion des candidats officiels, soit de la surveillance des adversaires. Même si certains agents concourent à la fois aux activités de promotion et de surveillance (c'est le cas des préfets lorsqu'ils se montrent loyaux vis-à-vis du gouvernement), ces deux réseaux sont, en pratique, le plus souvent distincts du fait de la présence simultanée, dans les différents départements, d'agents chargés de missions «électorales » et 
d'autres chargés de missions de surveillance. Ce réseau à géométrie variable n'est pas sans provoquer des tensions, par exemple entre des agents du ministère de la Police et les préfets de département ${ }^{9}$. En fait, cette architecture complexe, qui résulte de la division administrative entre ministère de l'Intérieur et ministère de la Police héritée $\mathrm{du}$ Directoire et de l'Empire ${ }^{10}$, correspond aussi à certaines contraintes de l'heure. Des membres de la noblesse d'Ancien régime, souvent de retour d'immigration et favorables aux députés sortants "ultra", occupent de nombreuses positions de pouvoir locaux, dans la magistrature ou dans l'armée. Pour les promoteurs de la dissolution, il est donc nécessaire de s'assurer de la loyauté de ces électeurs jugés influents et, dans le cas contraire, de s'assurer de l'envoi sur place d'agents susceptibles d'oeuvrer en leur lieu et place en faveur de la nomination des candidats officiels.

Tel est le cas dans le département de la Côte d'Or où le préfet, le Comte Hervé Clérel de Tocqueville ${ }^{11}$, soutient les députés sortants qui faisaient partie de la majorité de la "Chambre introuvable" tout en essayant de se faire nommer par le même groupe d'électeurs ${ }^{12}$. A cette occasion, il met à profit sa connaissance des "opinions" des maires et des fonctionnaires qu'il tente d'enrôler dans son entreprise de soutien aux candidats ultraroyalistes ${ }^{13}$. Le commissaire spécial envoyé dans ce département insiste auprès du ministre de la Police générale sur l'activité de ces derniers à la veille de la réunion du collège de département : "Il est probable maintenant que le préfet ne sera point élu. Trente électeurs se sont réunis le 2 [octobre 1816], et ont fait un scrutin particulier pour voir sur qui se porterait les votes. Excepté deux ou trois, tous ont nommé les trois ex-députés. Un de ces électeurs a dit que malgré le désir connu de M. de Tocqueville, il ne pensait pas qu'il fut nommé; que les anciens députés n'avaient en rien démérité de leurs concitoyens " ${ }^{14}$. Vraisemblablement renseigné par un des électeurs présent à cette réunion, le commissaire spécial ne se contente pas de fournir ces informations au comte Decazes si l'on en croit le compte-rendu qu'il fait de la réunion du collège électoral : «On a été obligé d'engager une partie des électeurs à se séparer pour que les anciens députés ne fussent pas réélus en corps $»^{15}$; parallèlement, il met l'accent sur le rôle des fonctionnaires publics ayant soutenu le camp adverse : «M. Riambourg, procureur général arrivant de Paris, a fait beaucoup de mal par ses visites à plusieurs fonctionnaires de l'ordre judiciaire et à des électeurs connus par leur modération, et qu'il a fait changer de sentiments [...] Les sous-préfets de Beaune et de Semur ont usé de toute leur influence en sens inverse de leurs devoirs $»^{16}$. Ces informations, soigneusement recoupées et rassemblées au sein du ministère, sont vraisemblablement à l'origine des changements intervenus peu après dans le personnel de l'administration préfectorale de ce département ${ }^{17}$. Ces prolongements de la crise de 1815-16 témoignent ainsi d'un lien entre le « circuit de surveillance " mis en place en 1816 et les pratiques administratives. C'est donc à partir de l'évolution des pratiques de la «candidature officielle» de leur reconduction à l'occasion de chaque nouvelle échéance électorale, que nous pouvons, à compter de cet épisode, comprendre les transformations des activités de surveillances.

Les transformation du « circuit de surveillance "

Dès les premières années de la Seconde Restauration, les pratiques de la « candidature officielle » imposent aux agents de l'administration de l'intérieur et de la police un effort de clarification de ce qui leur sert, sur le mode du sens pratique, de points de repère. "Le Gouvernement doit de bonne heure prendre les précautions nécessaires pour s'assurer l'influence supérieure dans les assemblées électorales, et n'avoir pour député que des propriétaires ou fonctionnaires dévoués au Roi et à son Gouvernement $»^{18}$. Cette sorte de gestion prévisionnelle, tenant compte des résultats des élections précédentes et de ce 
que les transformations de la législation électorale permettent d'espérer, aboutit à la simplification des "technologies d'Etat » mises en oeuvre en 1816 et au renforcement du rôle des préfets. Désormais, on "admet pour principe fondamental que les préfets de départements marchent toujours dans la ligne du Gouvernement ", car " on sent que s'il en était autrement, si quelques préfets se permettaient de sortir de cette ligne, pour servir tel ou tel parti, il y aurait alors absence de principes et de hiérarchie politique ${ }^{19}$. A la mesure des différentes échéances électorales qui se succèdent entre 1817 et 1820, c'est donc le «circuit de promotion » centré sur les préfets qui devient le point cardinal du répertoire d'action de la « candidature officielle».

Le rôle des préfets en matière électorale est bien connu dans ses grandes lignes, mais il est souvent mal apprécié ou surestimé : le travail historiographique reproduisant la "légende noire» des préfets du Second Empire et l'étendant volontiers à d'autres séquences historiques ${ }^{20}$. A cette époque, les préfets sont certes en mesure de contrôler l'ensemble des agents chargés d'activités de surveillance en lien avec les nominations à effectuer ${ }^{21}$. Mais ces pratiques sont loin d'être exclusivement liées au régime impérial ou aux contraintes qui résulteraient du suffrage universe ${ }^{22}$. C'est durant les premières années de la Seconde Restauration, à la faveur d'élections indécises et rapprochées, et dans le cadre naissant du répertoire d'action de la « candidature officielle ", que le rôle électoral des préfets s'accroît de manière significative.

Le «circuit de surveillance " évoqué dans la première partie est rapidement intégré dans un « circuit de promotion »au bénéfice des préfets qui n'ont plus à compter avec les commissaires extraordinaires ou avec les agents du ministère de la Police envoyés de Paris aux fins de superviser la nomination des députés. L'action des agents du ministère de la Police générale a eu un impact somme toute limité en 1816, et les préfets se sont révélés beaucoup plus efficaces dans leur capacité à mobiliser et à orienter le vote des électeurs censitaires, même si c'était parfois dans un sens peu conforme aux voeux des ministres et de l'ordonnance du 5 septembre. En effet, lorsqu'il s'agit de mobiliser et de rallier des électeurs appartenant largement à la noblesse et encore marqués par le "faire corps " qui sous-entendait les pratiques électorales d'Ancien régime, c'est moins la qualité des renseignements sur les personnes que la capacité à s'insérer dans un réseau de préséances et de hiérarchies sociales qui compte. $C^{\prime}$ est parce qu'ils sont en mesure de mettre en jeu leurs titres de noblesse que les préfets peuvent prétendre à une position centrale au sein des collèges électoraux ou, à défaut, seconder les présidents de collège dans leurs entreprises. Pour le dire autrement, la condition du succès électoral repose encore à cette époque sur des liens sociaux hérités de la société de cour, et de ses prolongements dans l'émigration, que cette forme de patronage d'Etat qu'est la «candidature officielle» doit prendre en compte.

C'est en fonction de la manière dont ces contraintes sont perçues en 1816, mais aussi des succès enregistrés par les candidats soutenus par les préfets, que ces derniers deviennent, dans les années qui suivent, les principaux maîtres d'oeuvre de la " candidature officielle ». Lors des renouvellements partiels et successifs de la Chambre des députés, les préfets se voient confier la tâche du recensement des "fonctionnaires publics, ou employés, qui peuvent influer sur les élections » avec mission de fournir au seul ministre de l'Intérieur "une notice confidentielle sur chacun " ${ }^{23}$. Dans ces conditions, la retenue conseillée aux préfets désireux d'être eux-mêmes candidats en 1816 n'est plus de mise ${ }^{24}$. Ce « circuit de nomination» raccourci où le préfet s'auto-désigne en quelque 
sorte comme candidat officiel a beaucoup d'avantages pour les gouvernements de la Restauration, car il simplifie les problèmes que pose toujours la sélection des candidats. De telles pratiques suscitent néanmoins des oppositions dans les rangs des parlementaires de la minorité ultraroyaliste. Plusieurs députés proposent le retour à un régime d'incompatibilité entre mandat électif et fonction publique. Seule l'incompatibilité relative des préfets et des commandants militaires dans les départements où ils exercent leurs fonctions leur est concédée (loi Laîné de 1817); mesure étendue aux sous-préfets en $1820^{25}$. Ces mesures auront peu d'effet: selon l'historien Nicolas Richardson, presque un quart des 164 préfets de la Restauration siègent à un moment ou à un autre sur les bancs de la Chambre des députés ${ }^{26}$.

Qu'en est-il alors des activités de surveillance ? La réunion des ministères de la Police et de l'Intérieur lors de la formation du cabinet Dessolle le 29 décembre 1818, avec la nomination de Decazes à ce poste, traduit de manière institutionnelle la subordination des activités de "basse police " à l'initiative des préfets et des sous-préfets ${ }^{27}$. C'est la sélection puis la promotion des "candidats officiels" qui constitue désormais l'essentiel des tâches préfectorales en matière électorale. Pour ce faire, ils s'appuient sur l'ensemble des fonctionnaires publics dont la loyauté électorale fait l'objet de la plus grande attention. Ceux-ci sont à la fois les acteurs et les objets des activités de surveillance liées aux pratiques électorales, qui dépassent en nombre la seule action des forces de police ou de gendarmerie. Qu'ils soient menacés de révocation ou désireux de faire carrière, les fonctionnaires électeurs ${ }^{28}$ se trouvent mobilisés en faveur des candidats officiels, et cette mobilisation donne naissance à un répertoire d'action nouveau où la nomination élective se défait peu à peu des habits d'Ancien régime qu'elle revêtait encore à l'occasion.

Quelle est la place des pratiques policières stricto sensudans ce répertoire ? Certes, il convient toujours de surveiller les adversaires des candidats officiels, ou leurs alliés peu sûrs, mais cette surveillance s'éloigne de plus en plus des pratiques policières ordinaires, telles les filatures ou le recours aux « mouches » (pratiques qui subsistent néanmoins) pour s'articuler à cette science des élections naissante dont les préfets, bien avant le Second Empire répétons-le, se font les inventeurs ${ }^{29}$. Outre les informations standardisées demandées par voie de circulaires par le ministère de l'Intérieur, les préfets ou sous-préfets accumulent parfois pour eux-mêmes des renseignements sur les électeurs de leurs départements ou de leurs arrondissements. Ce travail de renseignement que révèle le carnet tenu par le sous-préfet d'Apt, Charles de Martignan ${ }^{30}$, en 1824, a désormais une finalité essentiellement électorale: «Il ne s'agissait pas seulement de noter l'appréciation des caractères,note l'historien qui a édité ces carnets: la nécessité s'imposait d'indiquer les moyens propres à entraîner, à circonvenir l'électeur, à l'obliger même en cas de besoin, à formuler un vote favorable [...] tout est mis en oeuvre, les simples politesses, les invitations à dîner, les influences des amis, des parents, $d u$ clergé, du préfet et du ministre, l'argent, l'ambition, et la crainte de la justice ${ }^{31}$.

Cet exemple, loin d'être un cas isolé, montre bien comment les activités de surveillance sont à la fois constitutives des pratiques de la «candidature officielle» et, dans le même temps, sont rendues possibles par les contraintes électorales que font peser ces dernières sur les agents de l'administration. "Faire voter » et «faire élire », ce n'est pas uniquement du travail de "basse police", néanmoins les activités de promotion et de mobilisation électorale des agents de l'administration ne sont jamais pour autant 
très éloignées des pratiques de surveillance et de renseignement qui caractérisent alors (et sans doute encore aujourd'hui) le travail de police.

A partir de ces éléments d'analyse circonstanciés, il est possible d'élaborer des hypothèses de portée plus générale afin, non pas de clore le sujet, mais de nourrir la discussion entre spécialistes de différentes disciplines dans le cadre de recherches sur la genèse des pratiques et des dispositifs de surveillance.

L'intensité de la surveillance politique en matière électorale - qui progresse de manière significative sous le Second Empire, entre autre du fait de l'augmentation du nombre de fonctionnaires spécialisés dans les activités de police ${ }^{32}$ - est liée aux investissements d'agents de l'Etat dans les opérations électorales à travers les différentes formes de candidatures recommandées, des "candidatures ministérielles» de la monarchie censitaire aux « candidatures officieuses » de la Troisième République après $1877^{33}$.

$\mathrm{Au}$ sein du répertoire d'action de la "candidature officielle », le travail de prévision n'est jamais séparé du travail de surveillance. Cette mise en forme pratique de savoirfaire administratifs a survécu à l'euphémisation et à la disparition au XXème siècle des pratiques de la "candidature officielle", notamment à travers la formation d'une bureaucratie spécialisée à partir du service des renseignements généraux créé en 1894 et associant étroitement ces deux types d'activités ${ }^{34}$. En fonction des éléments historiographiques disponibles - l'histoire comparée de la construction de l'opération électorale dans l'ensemble des Etats parlementaires étant loin d'être achevée ${ }^{35}$ - cette longévité des pratiques de "surveillance politique" semble une caractéristique singulière des pratiques électorales françaises...

Ces deux hypothèses de travail nous renvoient aux problèmes évoqués dans l'introduction de cet article : à quel degré et à quel type de spécialisation des pratiques de surveillance fait-on référence lorsque l'on parle de "surveillance politique »? Répondre de manière plus détaillée à cette question, ce qui suppose d'appréhender dans sa complexité le mouvement d'autonomisation des pratiques politiques tout au long du XIXème siècle, devrait permettre d'évaluer dans quelle mesure ces pratiques de surveillance sont constitutives d'un ordre politique qui prend aujourd'hui (mais pas nécessairement de manière ultime) la forme d'un ordre démocratique et dans lequel la construction de l'opération électorale comme élection libre et concurrentielle joue un rôle décisif.

\section{NOTES}

1. . Corbin A., Le monde retrouvé de Louis-François Pinagot. Sur les traces d'un inconnu, 1798-1876, Paris, Ed. Flammarion, « Champs », 2002 (1ère ed. 1998), p. 248.

2. . Sur ces obstacles à l'analyse, voir Lacroix B., « Ordre politique et ordre social. Objectivisme, objectivation et analyse politique », in Grawitz M., Leca J., (dir.), Traité de science politique, Paris, Presses Universitaires de France, 1985, vol. 1, pp. 469-565.

3. . Voir, à titre d'exemple, Bigo D., Polices en réseaux. L'expérience européenne, Paris, Presses de Sciences Po, 1996, 343 p. 
4. . Que l'on peut définir comme l'ensemble des transactions entre agents mobilisés pour l'obtention de charges électives qui fonctionnent comme un mécanisme de « concentration de capital symbolique » - l'expression est empruntée à Pierre Bourdieu - à travers un travail de mobilisation relatif à l'opération électorale et qui donne ainsi naissance à un répertoire d'action spécifique.

5. . Sur le contexte de la crise des années 1815 et 1816 et plus généralement sur les pratiques de la " candidature officielle », voir Voilliot Ch., La candidature officielle en France de la Restauration aux débuts de la Troisième République. Retour sur l'historiographie d'une pratique d'Etat, Thèse de science politique sous la direction de B. Lacroix, Université de Paris-X Nanterre, 2002, ch. 1.

6. . Outre une bonne partie des originaux des rapports lorsqu'ils étaient adressés directement au ministre de la Police et des copies de la correspondance saisie, les Archives nationales ont conservé, sous les cotes F7 4348 à 4352, deux cahiers manuscrits : le premier contient la synthèse de 600 rapports, notes ou lettres concernant tous les départements sauf celui de la Seine ; le second, les résultats des différents collèges. Sur ces 600 documents exploités, 264 proviennent des préfets, 219 de particuliers, 35 des lieutenants ou commissaires de police, 54 des commissaires spéciaux, 22 des présidents de collège et 6 sont d'origines diverses. En raison d'un classement hasardeux, ces documents ont été confondus dans les inventaires des Archives nationales avec d'autres documents de même origine et de même nature, mais concernant les élections de 1818.

7. . Il n'est pas possible de fournir une liste précise de ces agents mobilisés (encore moins un organigramme de ce circuit). Les statuts, les rôles et les fonctions précises de ces agents varient d'un département à l'autre. Cela va du préfet, représentant officiel du gouvernement, aux officiers de police et de gendarmerie, en passant par toute la panoplie des « mouches » et des collaborateurs plus ou moins spontanés...

8. . Le ministre de l'Intérieur, Laîné, adopte à cette occasion un comportement plus prudent : " S'il juge les ultras excessifs et dangereux, il refuse cependant d'entrer en rupture ouverte avec eux par crainte de livrer la monarchie clés en main aux libéraux ». De Waresquiel E., Yvert B., Histoire de la Restauration, 1814-30. Naissance de la France moderne, Paris, Librairie Académique Perrin, 1996, p. 189.

9. Dans la Nièvre, le préfet De Vaines, beau-frère de Guizot, se plaint auprès de Decazes, ministre de la Police générale, des missions d'un dénommé RousseauChatillon. Voir la correspondance publiée par Thuillier G., « Les rapports d'un agent secret sur Clamecy et son arrondissement en 1816 », Bulletin de la société scientifique et artistique de Clamecy, 2000, pp. 85-93.

10. . Inauguré par la loi du 12 nivôse an IV (2 janvier 1796) créant un ministère de la Police générale, ce dédoublement qui concernait les ministères les plus importants avait pour objectif de favoriser le contrôle de l'Empereur sur ses ministres en évitant une répartition à priori des compétences et en créant les conditions d'une concurrence institutionnelle entre les titulaires des différentes fonctions. De ce fait, à partir de 1808-09, les ministres sont plus étroitement soumis au despotisme impérial. A ce sujet, voir Petiteau N., Napoléon. De la mythologie à l'histoire, Paris, Ed. du Seuil, « L'univers historique ", 1999, p. 224 et s.

11. . Né à Menou dans la Nièvre le 3 août 1772, devenu préfet à la faveur de la Restauration, il est nommé en Côte d'Or le 31 janvier 1816. Archives nationales F1bI $174 / 9$. 
12. . Pour une présentation plus détaillée des luttes politiques dans ce département, voir Fizaine S., La vie politique en Côte d'or sous Louis XVIII. Les élections et la presse, Dijon, Publications de l'université, 1931, 272 p. ; Gonnet P., La société dijonnaise au XIXème siècle, Thèse d'histoire, Université de Paris I, 1974.

13. . Lors de la tournée qui suit sa nomination, il reçoit tous les maires aux chefs-lieux de canton et note dans son journal le nom des fonctionnaires qui lui paraissent « influents en mauvais sens ». Voir Gaveau F., «L'Etat et l'écoulement des Temps sous la Restauration. La hantise de l'événement ", Annales de Bourgogne, LXXIV, 1, janvier-mars 2002, pp. 89-90.

14. . Rapport du commissaire spécial du 3 octobre 1816. Archives Nationales F7 3451A dossier 1 historique.

15. . L'expression témoigne de la prégnance des modes de faire et de penser de l'Ancien régime. Cette tactique, pratiquée dans plusieurs collèges par les deux « camps » en présence fut parfois à l'origine d'incidents empêchant la nomination des députés. Voilliot Ch., « Les incidents électoraux et la contestation des pratiques de la candidature officielle en 1816 ", in Bourdin Ph., Caron J.Cl., Bernard M., (dir.), L'incident électoral de la Révolution française à la Vème République, Clermont-Ferrand, Presses Universitaires Blaise-Pascal, « Histoires croisées », 2002, pp. 88-99.

16. Rapport du commissaire spécial du 4 octobre 1816. Archives Nationales F7 3451A dossier 1 historique.

17. . Le préfet, Hervé Clérel de Tocqueville, est remplacé le 19 février 1817 et est nommé ensuite dans le département de la Moselle. Le sous préfet de Beaune, Etienne Perrin du Lac, est révoqué le 19 mars 1817.

18. . Quelques observations sur le système général des élections dans un gouvernement représentatif, adaptées à la situation actuelle de la France - Archives Nationales F7 3451A. Note rédigée en 1818 pour le ministre de la Police générale.

19. Ibid.

20. . A l'inverse, des travaux récents de science politique tendent à minimiser leur rôle en matière électorale. Voir Guionnet C., L'apprentissage de la politique moderne. Les élections municipales sous la Monarchie de Juillet, Paris, Ed. L'Harmattan, « Logiques politiques ", 1997.

21. . Voir l'ouvrage classique de Le Clère B., Wright V., Les préfets du Second Empire, Paris, Armand Colin, 1973, 411 p. ; et la mise au point récente de Price R., The French Second Empire. An Anatomy of Political Power, Cambridge, Cambridge University Press, 2001, pp. 95-133.

22. . Sur ce dernier point, voir Quéro L., Voilliot Ch., « Du suffrage censitaire au suffrage universel. Evolution ou révolution des pratiques électorales? ", Actes de la recherche en sciences sociales, 140, décembre 2001, pp. 34-40.

23. . Circulaire confidentielle aux Préfets du 27 mars 1818. Archives Nationales F1cII 49. 24. . Voilliot Ch., La candidature officielle, op. cit. , ch. 3.

25. . La proposition de De Conny, député ultraroyaliste de l'Allier, du 12 mars 1828 visant à soumettre à réélection « tout membre de la Chambre des députés qui sera élevé à de nouvelles fonctions » est repoussée par la Chambre des pairs à la suite des conclusions négatives du rapporteur Pasquier. Pour un résumé de ces débats, voir Girard L., « La réélection des députés promus à des fonctions publiques, 1828-31 », in La France au XIXème siècle. Mélanges offerts à Charles-Hyppolyte Pouthas, Paris, Publications de la Sorbonne, «Etudes », 1973, pp. 227-244. 
26. . "The extension of this personal patronage was one that was purely political [...] Excluding the small number of prefects, like Boula de Colombiers whose administrative career was over before they became deputies, the remainder fall into two fairly evenly divided classes : the prefects who were subsequently made deputies while still continuing their careers in the administration, and the deputies who where simultaneously or subsequently given prefectures. In both cases, the political justification is clear. A prefect elected as deputy meant a vote the minister could justifiably regard as automatic, the prefecture acting as pledge. The deputy made prefect had often given his guarantee already, and the prefecture was a reward conditional on his continued fidelity ». Richardson N., The French Prefectoral Corps, 1814-1830, Cambridge, Cambridge University Press, 1966, p. 37. Voir aussi Machin H., The Prefect in French Public Administration, Londres, Croom Helm, 1977, p. 23 et suivantes.

27. . Riberette P., « De la police de Napoléon à la police de la Congrégation », in Aubert J., et al, L'Etat et sa police en France, 1789-1914, Genève, Librairie Droz, « Hautes études médiévales et modernes ", 1979, pp. 35-58.

28. . Ils forment souvent à eux seuls une majorité au sein des collèges électoraux des arrondissements ruraux.

29. . Phélippeau E., « La fabrication administrative des opinions politiques », Revue française de science politique, XXXXIII, 4, août 1993, pp. 587-612 ; Ibid., " Conjonctures électorales et conjonctures préfectorales. Le vote et la formation d'un savoir-faire politico-administratif », Scalpel. Cahiers de sociologie politique de Nanterre, 1, 1994, pp. 52-73.

30. . Né le 1er avril 1762, nommé sous-préfet de Forcalquier en août 1815, il est remplacé en 1819. Nommé à Apt en 1822, il y demeure jusqu'à la Révolution de juillet 1830. Archives Nationales F1bI 167/9.

31. Sauvé F., Les dessous d'une élection législative en province en 1824 (d'après le carnet d'un sous-préfet d'Apt), St-Amand, Pivoteau, 1904, p. 13.

32. . Voir, à titre d'exemple, Ebel E., Police et société. Histoire de la police et de son activité en Alsace au XIXème siècle, Strasbourg, Presses Universitaires de Strasbourg, 2000.

33. L'expression désigne des candidatures recommandées discrètement et sans qu'il soit fait appel de manière systématique à des « technologies d'Etat » disqualifiées sur le plan électoral dans leur usage ostentatoire, mais toujours présentes à l'état de tentations pratiques, quoique engagées par le répertoire codifié qui définit désormais la légitimité « démocratique » de l'opération électorale. Cf. Voilliot Ch., La candidature officielle, op. cit. , 3ème partie.

34. . Dewerpe A., « La République a-t-elle besoin d'espions ? ", in Baruch M.O., Duclert V., (dir.), Serviteurs de l'Etat. Une histoire politique de l'administration française, 1875-1945, Paris, Ed. La Découverte, 2000, p. 149.

35. . Pour un premier bilan, voir Noiret S. (dir.), Political Strategies and Electoral Reforms : Origins of Voting Systems in Europe in the 19th and 20th Centuries, Baden Baden, Nomos Verlagsgesellschaft, 1990. Voir également « La campagna elettorale nell'Europa mediterranea ", Memoria e Ricerca. Rivistia di storia contemporanea, 8, juillet-décembre 2001. 
INDEX

Index géographique : France

Mots-clés : démocratie, surveillance 